conjecture that the number was 1 for all values of $N$. A precisely equivalent conjecture was the basis for a spurious proof of Fermat's last theorem announced by Lamé and Cauchy in 1846, and refuted by Kummer's revelation that factorization into primes is not unique in the cyclotomic integers of degree 23.

For $N=46$, Kummer's discovery means that there are three distinct lattices, two of them, crystallographers will be astonished to learn, constituting an enantiomorphic pair. After that things become even wilder. There are, for example 359,057 distinct lattices with 128 -fold symmetry. The computation of numbers like this is a highly non-trivial exercise, but fortunately mathematicians have been working on the problem for 140 years (it is still not completely solved), kindly saving quasicrystallographers a lot of hard work.

It is remarkable that after almost a century and a half of pristine purity, this branch of mathematical research should have found two utterly unrelated physical applications within a single year.

Laboratory of Atomic and

N. David Mermin

Solid State Physics,

Cornell University,

Ithaca,

New York 14853, USA

\section{The 'last ribo-organism' was no breakthrough}

SrR-Benner and Ellington ${ }^{1}$ emphasize the important point that primordial riboorganisms made almost entirely of RNA and living in the RNA world ${ }^{2}$ might have evolved relatively sophisticated forms of intermediary metabolism before the advent of protein synthesis. Unfortunately, the authors arrive at this correct conclusion by an argument that reveals a profound misunderstanding of basic chemistry and biology, as well as current ideas about molecular evolution.

Like others before them ${ }^{2.3}$, Benner and Ellington are struck by the ubiquitous role in present-day intermediary metabolism of nucleotide cofactors such as FAD, NAD, S-adenosyl methionine, CoA and coenzyme $\mathbf{B}_{12}$. We fail to understand, however, why they believe the existence of nucleotide cofactors to be evidence for metabolic complexity in the RNA world before the advent of protein synthesis. As the side chains of amino acids are chemically limited, nucleotides are far better catalysts than proteins for many reactions, particularly those involving oxidation/ reduction. Thus, evolving ribonucleoprotein (RNP) or protein enzymes might have adopted nucleotide cofactors de novo, regardless of whether the RNA world was metabolically simple or complex.

Benner and Ellington fall into the trap of believing that any organism as adapt- able as a virus must be completely modern because it is the product of constant streamlining. This leads them to ridicule our 'genomic tag' model for the origin of protein synthesis ${ }^{4.5}$ on the grounds that it is "unlikely that RNA viruses living in modern hosts contain many nonfunctional vestiges of an RNA world that vanished 2,000 million years ago".

As recently discussed in a News and Views article ${ }^{6}$, we proposed in our model that the $3^{\prime}$-terminal tRNA-like structures of contemporary bacterial and plant RNA viruses may be direct descendents of $3^{\prime}$ terminal genomic structures in the RNA world ${ }^{4.5}$. This hypothesis provides the first plausible pathway for the stepwise evolution of protein synthesis, as tRNAlike structures (and perhaps specific aminoacylation) would have evolved as part of the replication machinery before the advent of protein synthesis. We also argued that several aspects of contemporary tRNA metabolism can be quite rigorously and consistently viewed as 'molecular fossils' - reactions or pathways that were used in the RNA world and persist today. The 3 '-terminal tRNA-like structures are still functional today, nor would they be preserved if they were not. And the biochemistry to which we have applied the term molecular fossil is all contemporary and essential; we believe it was equally essential several thousand million years ago.

Benner and Ellington confuse the concept of a molecular fossil ${ }^{4.5}$ with the vernacular notion of a living fossil. For example, the fact that all organisms synthesize DNA precursors by reducing RNA precursors (using the enzyme ribonucleoside diphosphate reductase) is commonly interpreted as a molecular fossil, indicating that the pathways for synthesis of RNA evolved before the need for $\mathrm{DNA}^{7}$. These pathways persist because it is too late to change them; the pathways are functional, the products are essential, and the individual reactions are subject to complex regulation. Living fossil, on the other hand, is a casual term for any organism whose survival we find surprising. Living fossils are largely quirks of fate; molecular fossils survive because they are essential.

Contrary to the suggestion that many of us who think seriously about early evolution believe that the era during which RNA served as the principal catalyst in living systems was necessarily short because proteins are more versatile catalysts than RNA, neither we, nor any of the other authors cited by Benner and Ellington, have ever dared to estimate the longevity of the RNA world. We also fail to understand why any attempt to understand the origin of protein synthesis must be construed as underestimating the abilities of catalytic RNA. Our proposal that the first aminoacyl-tRNA synthetases were made of RNA $^{4,5}$ is clearly an affirmation of metabolic diversity and complexity in the RNA world, and we are surprised that Benner and Ellington misinterpret this proposal as revealing a mental fixation on the catalytic power of proteins.

Finally, Benner and Ellington make the serious error of assuming that the remarkable complexity of modern protein synthesis arose essential all at once, rather than one tiny step at a time. This point of view leads them to postulate a 'breakthrough' organism - the first riboorganism to invent protein synthesis. For all its rhetorical flair, the notion of a breakthrough organism is intellectually insidious because it confuses two very different ideas - one of them obvious, the other wrong.

The obvious idea is that all forms of life on Earth must have descended from a single common ancestor or progenote ${ }^{8}$; no other conclusion is consistent with the remarkable similarity of all cells at the biochemical level. The wrong idea is that this progenote (reborn as the breakthrough organism?) survived because it alone was clever enough to invent protein synthesis. We prefer the more biologically plausible view that the RNA world evolved imperceptibly into an RNP world, with many interbreeding organisms contributing to the invention of protein synthesis as we know it. Whether the particular organism that we now call the progenote survived because it was better at making protein than its fellows, or simply because it did not have the bad luck to be burnt to a crisp in a volcanic eruption or swallowed up in an antedeluvian deluge, is not something we are ever likely to know.

NANCY MaIzels Alun M. WeINER

Department of Molecular Biophysics and Biochemistry,

Yale University,

333 Cedar Street, New Haven,

Connecticut 06510, USA

Benner, S.A. \& Ellington, A.D. Nature 329, 295-296 (1987)

2. Gilbert, W. Nature 319,618 (1986)

3. White, H.B. III J. molec. Evol. 7, 101 (1976).

4. Weiner, A.M. \& Maizels, N. Proc. natn. Acad. Sci. U.S.A. 84, 7383-7387 (1987).

5. Maizels, N. \& Weiner, A.M. Cold Spring Harb. Symp. quant. Biol. (in the press)

6. North, G. Nature 328, 18 19 (1987).

7. Watson, J.D. et al. Molecular Biology of the Gene 4th edn, Vol. II, Ch. 28 (Benjamin Cummings, Menlo Park, 1987). 8. Pace, N.R., Olsen, G. \& Woese, C.R. Cell 45, 325-326 (1986).

\section{Scientific Correspondence}

Scientific Correspondence is intended to provide a forum in which readers may raise points of a scientific character They need not arise out of anything published in Nature. In any case, priority will be given to letters of less than 500 words and five references. 